\title{
Effect of lipids on rumen fermentation and kinetics of rumen digesta in sheep fed a restricted diet *
}

\author{
C Van Nevel, D Demeyer, S De Smet
}

Research Centre for Nutrition, State University Gent, 9090 Melle, Belgium

\begin{abstract}
Soya oil hydrolysate has been found to decrease the fractional outflow rate of solids in the sheep rumen (Broudiscou et al, 1990). In this study, the effect of soya oil (SO) and tallow (TA) on rumen fermentation and on liquid and particle outflow rates and volumes has been investigated.
\end{abstract}

Three rumen-cannulated sheep were fed (latinsquare design) the following rations: BR $(300 \mathrm{~g}$ concentrates and $300 \mathrm{~g}$ hay administered at $8.30 \mathrm{~h}$ and $20.30 \mathrm{~h}$ ); SO (BR with $40 \mathrm{~g}$ SO twice daily) or TA (BR with $40 \mathrm{~g}$ TA twice daily). Fractional outflow rates of rumen liquid (D) and particles $(\mathrm{K})$ were measured (twice/animal/ration) using PEG or Cr-mordanted hay. Rumen liquid volume (V) and dry matter pool (M) were calculated from the marker dilution curves. Fermentation pattern and protozoal counts ( 3 times/ animal/ration) and in sacco degradation of soya bean meal (incubation time: $6 \mathrm{~h}$ ) and hay (inc ubation time: 24 h) (4 times/animal/ration) were also determined.

Table I shows some of the results. Molar percentages of propionate were significantly increased while butyrate percentages decreased, but not in a statistically significant manner, whilst $\mathrm{pH}$ remained unchanged.

Total rumen volatile fatty acids (VFA) were decreased by $\mathrm{SO}$ and rumen $\mathrm{NH}_{3}-\mathrm{N}$ concentration by $\mathrm{SO}$ and TA. Apart from differences in absorption (unlikely), the latter is probably associated with decreased protozoal numbers while the former must be related to lower in sacco degrabilities and increase in rumen particle pool size and outflow, according to models of rumen bacterial growth and digestion. Lower protozoal numbers were indeed associated with higher values for microbial protein synthesis and its efficiency ( $\mathrm{g} \mathrm{N}$ incorporated per $\mathrm{kg}$ organic matter fermented) (Sutton et al, 1983).

Broudiscou L, Van Nevel CJ, Demeyer DI (1990) Anim Feed Sci Technol 30, 51-67

Sutton JD, Knight $R$, McAllan $A B$, Smith $R H$ (1983) Br J Nutr 49, 419-432

Table I. Effect of lipids on rumen fermentation and kinetics.

\begin{tabular}{|c|c|c|c|c|c|c|c|c|c|}
\hline \multirow[b]{2}{*}{ Ration } & \multirow[b]{2}{*}{$\underset{(\mathrm{mmol} /)}{\sum V F A}$} & \multirow[b]{2}{*}{$\begin{array}{l}\mathrm{NH}_{3}-\mathrm{N} \\
(\mathrm{mg} / \mathrm{l})\end{array}$} & \multirow[b]{2}{*}{$\begin{array}{c}D \\
(\% / h)\end{array}$} & \multicolumn{2}{|c|}{ Kinetics } & \multicolumn{4}{|c|}{ Degraded in sacco } \\
\hline & & & & $\begin{array}{l}V \\
(I)\end{array}$ & $\begin{array}{c}k \\
(\% / h)\end{array}$ & $\begin{array}{l}M \\
(g)\end{array}$ & $\begin{array}{c}\text { Soya-TN } \\
(\%)\end{array}$ & $\begin{array}{c}\text { Hay-NDF } \\
(\%)\end{array}$ & $\begin{array}{l}\text { Protozoa } \\
\times 10^{6} / \mathrm{mI}\end{array}$ \\
\hline BR & $91^{a}$ & $129^{a}$ & $7.4^{a}$ & $8.7^{a}$ & $3.4^{\mathrm{a}}$ & $1232^{a}$ & $29.3^{a}$ & $39.7^{a}$ & $1.01^{\mathrm{a}}$ \\
\hline SO & $75^{b}$ & $65^{b}$ & $7.3^{a}$ & $9.2^{\mathrm{a}}$ & $3.9^{a}$ & $1287^{a}$ & $36.3^{a}$ & $36.8^{a}$ & $0.61^{a}$ \\
\hline TA & $84^{a b}$ & $66^{b}$ & $8.0^{\mathrm{a}}$ & $8.9^{a}$ & $3.8^{a}$ & $1282^{a}$ & $33.2^{a}$ & $35.2^{\mathrm{a}}$ & $0.82^{a}$ \\
\hline RSD & 0.5 & 2 & 1.7 & 0.8 & 0.0 & 255 & 11.4 & 8.9 & 0.21 \\
\hline
\end{tabular}

$V, M, T N$, NDF: respectively liquid volume, dry matter pool, total nitrogen, neutral detergent fibre. $a, b: P<0.05$; SVFA: sum of the volatile fatty acids.

*Sponsored by IWONL, Brussels. 